\title{
Rosuvastatin protects PC12 cells from hypoxia/reoxygenation-induced injury by inhibiting endoplasmic reticulum stress-induced apoptosis
}

\author{
YU GAO, LIBO LI, JIANBAI YU and ZHANWEI ZHANG \\ Department of Neurosurgery, First Affiliated Hospital of Hunan University of \\ Traditional Chinese Medicine, Changsha, Hunan 410007, P.R. China
}

Received December 29, 2020; Accepted April 23, 2021

DOI: $10.3892 /$ etm.2021.10623

\begin{abstract}
The endoplasmic reticulum stress (ERS) response serves an important role in cerebral ischemia-reperfusion injury (CIRI). However, to the best of the our knowledge, the effect of rosuvastatin on the ERS response in CIRI has not yet been studied. In the present study, the effect of rosuvastatin on cell damage in CIRI was investigated; furthermore, the effect of rosuvastatin on the ERS response was explored. Firstly, a hypoxia/reoxygenation (H/R)-induced cell damage model was established in PC12 cells. Cell viability was subsequently detected by a Cell Counting Kit- 8 assay. A lactate dehydrogenase kit was used to detect cytotoxicity. TUNEL assay was then used to measure the extent of cell apoptosis, and western blotting was used to analyze the expression levels of the apoptosis-associated proteins Bax, Bcl-2, cleaved caspase- 3 and cleaved caspase- 9 . In addition, western blotting was used to detect the expression levels of ERS-associated proteins, including phosphorylated (p)-protein kinase R-like endoplasmic reticulum kinase (PERK), p-eukaryotic initiation factor $2 \alpha$ and other proteins. Treatment with rosuvastatin led to an increased activity of H/R-induced PC12 cells and a decrease in their cytotoxicity. Rosuvastatin also led to an inhibition in apoptosis and ERS in H/R-induced PC12 cells. After administration of the ERS response activator thapsigargin (TG), TG was found to reverse the protective effect of rosuvastatin on injury of H/R-induced PC12 cells. Taken together, these findings have shown that rosuvastatin is able to protect PC12 cells from H/R-induced injury via inhibiting ERS-induced apoptosis, providing a strong theoretical basis for the use of rosuvastatin in the clinical treatment of CIRI.
\end{abstract}

Correspondence to: Dr Zhanwei Zhang, Department of Neurosurgery, First Affiliated Hospital of Hunan University of Traditional Chinese Medicine, 95 Shaoshan Middle Road, Changsha, Hunan 410007, P.R. China

E-mail: gaoyugyyg@163.com

Key words: rosuvastatin, cerebral ischemia-reperfusion injury, endoplasmic reticulum stress, apoptosis, $\mathrm{PC} 12$ cells

\section{Introduction}

Cerebral ischemia-reperfusion injury (CIRI) refers to an acute cerebral dysfunction that occurs after cerebral ischemia for a certain period of time, followed by the restoration of the infarction-associated blood supply (1). The cure rate associated with CIRI is very low, accompanied by a high disability rate and a high mortality rate; and the prognosis is very poor, with the condition seriously affecting individual's health (2). CIRI is a complex multi-factorial process whose underlying mechanism is poorly understood. The current consensus is that post-ischemia reperfusion causes pathophysiological cascade reactions, including the explosive release of intracellular $\mathrm{Ca}^{2+}$ ions, excessive production of reactive oxygen species and neutrophil recruitment, which in turn leads to an acceleration of the inflammatory process, sustains ischemia and causes cell damage (3). The underlying pathological mechanisms include energy metabolism disorder, the inflammatory response, oxidative stress, excitatory amino acid toxicity and apoptosis (4).

Recently, a large number of studies have shown that apoptosis induced by endoplasmic reticulum stress (ERS) fulfills an important role in CIRI (5-7). ERS occupies a central role in CIRI and can initiate neuronal apoptosis (8). Hypoxia, energy depletion, acidosis, damage to $\mathrm{Ca}^{2+}$ homeostasis and the generation of a large number of free radicals caused by ischemia and reperfusion can all induce ERS, and stress-protective effects in cells may be generated in cells by ERS itself. However, excessive or persistent ERS is able to activate apoptotic signaling pathways, induce apoptosis and aggravate CIRI $(9,10)$. A previously published study reported that the ER-associated protein reticulon 1 is able to mediate CIRI through ERS and mitochondria-associated apoptotic pathways (11). In addition, a vascular endothelial growth factor antagonist was demonstrated to attenuate ischemic/reperfusion (I/R)-induced injury through inhibiting ERS-mediated apoptosis (12). Therefore, an increasing body of evidence supports that ERS has an important role in CIRI.

Among the therapeutic drugs that are currently available for cerebral infarction, statins are often used for primary and secondary prevention, as these have been shown to be important drugs for the treatment of cerebral infarction and to significantly decrease the risk of patients with cerebral infarction (13). In 
A<smiles>CC(C)c1nc(N(C)S(C)(=O)=O)nc(-c2ccc(F)cc2)c1/C=C/C(O)CC(O)CC(=O)O</smiles>

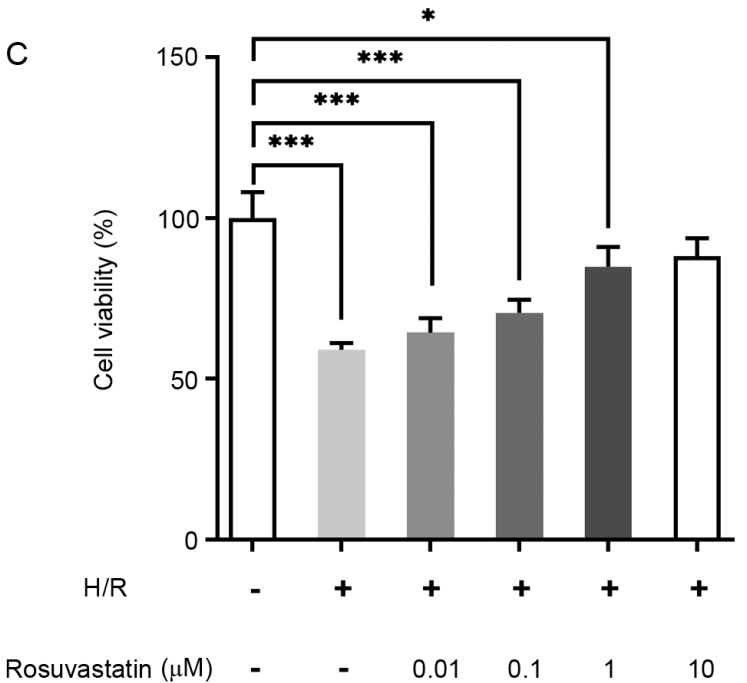

B
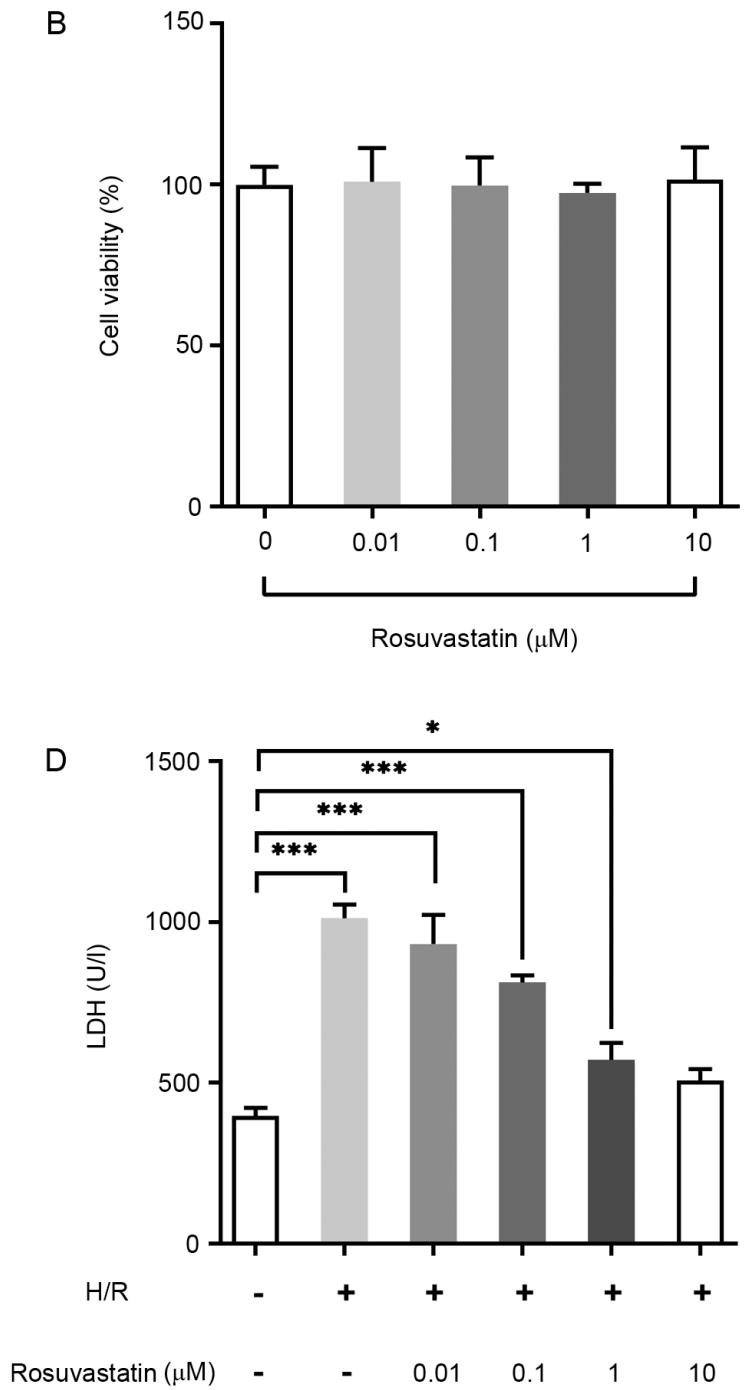

Figure 1. Rosuvastatin inhibits decreased cellular activity in H/R-induced PC12 cells. (A) Chemical formula of rosuvastatin. (B) CCK-8 detected the cell viability of PC12 cells after induction with different concentrations of rosuvastatin. (C) CCK-8 detected the cell viability of H/R-induced PC12 cells after induction with different concentrations of rosuvastatin. (D) LDH kit detected the cytotoxicity. ${ }^{*} \mathrm{P}<0.05 ;{ }^{* * * *} \mathrm{P}<0.001$. CCK-8, Cell Counting Kit-8; H/R, hypoxia/reoxygenation; LDH, lactate dehydrogenase.

addition to its powerful lipid-lowering effects, rosuvastatin is also useful in terms of its anti-inflammatory, antioxidant and immunological regulation effects, and for its ability to improve vascular endothelial function (14). A previous study revealed that rosuvastatin in combination with resveratrol is able to protect the synergic nerve in cases of CIRI (15). Rosuvastatin preconditioning exerts a marked protective effect on focal CIRI in rats, and its mechanism of action is predominantly to decrease oxidative stress and the inflammatory response (16). Rosuvastatin in combination with other drugs has been shown to decrease the size of cerebral infarction (17). In addition, it has been reported in other diseases that rosuvastatin may protect umbilical venous endothelial cells from damage induced under high-glucose conditions by decreasing ERS (18). Rosuvastatin was also shown to inhibit the apoptosis of endothelial cells in $\mathrm{ApoE}^{-/-}$mice via inhibiting ERS, and through the alleviation of atherosclerosis (19). However, to the best of our knowledge, the effect of rosuvastatin on ERS in CIRI has not yet been reported.

In the present study, PC12 cells were induced to establish a cell damage model by hypoxia/reoxygenation $(\mathrm{H} / \mathrm{R})$; the apoptosis of the model cells was subsequently detected after administering rosuvastatin, and the underlying mechanism was investigated.

\section{Materials and methods}

Cell culture and model induction. PC12 cells were obtained from the American Type Culture Collection and cultured in Dulbecco's Modified Eagle Medium (Gibco; Thermo Fisher Scientific, Inc.) with $10 \%$ fetal bovine serum (Gibco; Thermo Fisher Scientific, Inc.). The PC12 cells were cultured at $37^{\circ} \mathrm{C}$ in an atmosphere containing $5 \% \mathrm{CO}_{2}$ in a humidified incubator. For induction of the $\mathrm{H} / \mathrm{R}$ model, after culture at $37^{\circ} \mathrm{C}$ with $5 \% \mathrm{CO}_{2}$ for $24 \mathrm{~h}, \mathrm{PC} 12$ cells were placed under hypoxic conditions $\left(3 \% \mathrm{O}_{2} / 5 \% \mathrm{CO}_{2} / 92 \% \mathrm{~N}_{2}\right)$ for $2 \mathrm{~h}$. The cells were then cultured under normal conditions for $12 \mathrm{~h}$. In terms of cell administration, PC12 cells were pretreated with different concentrations of rosuvastatin $(0.01,0.1,1$ and $10 \mu \mathrm{m}$, cat. no. HY-17504A, MedChemExpress) for $12 \mathrm{~h}$ at $37^{\circ} \mathrm{C}$ before model induction. 

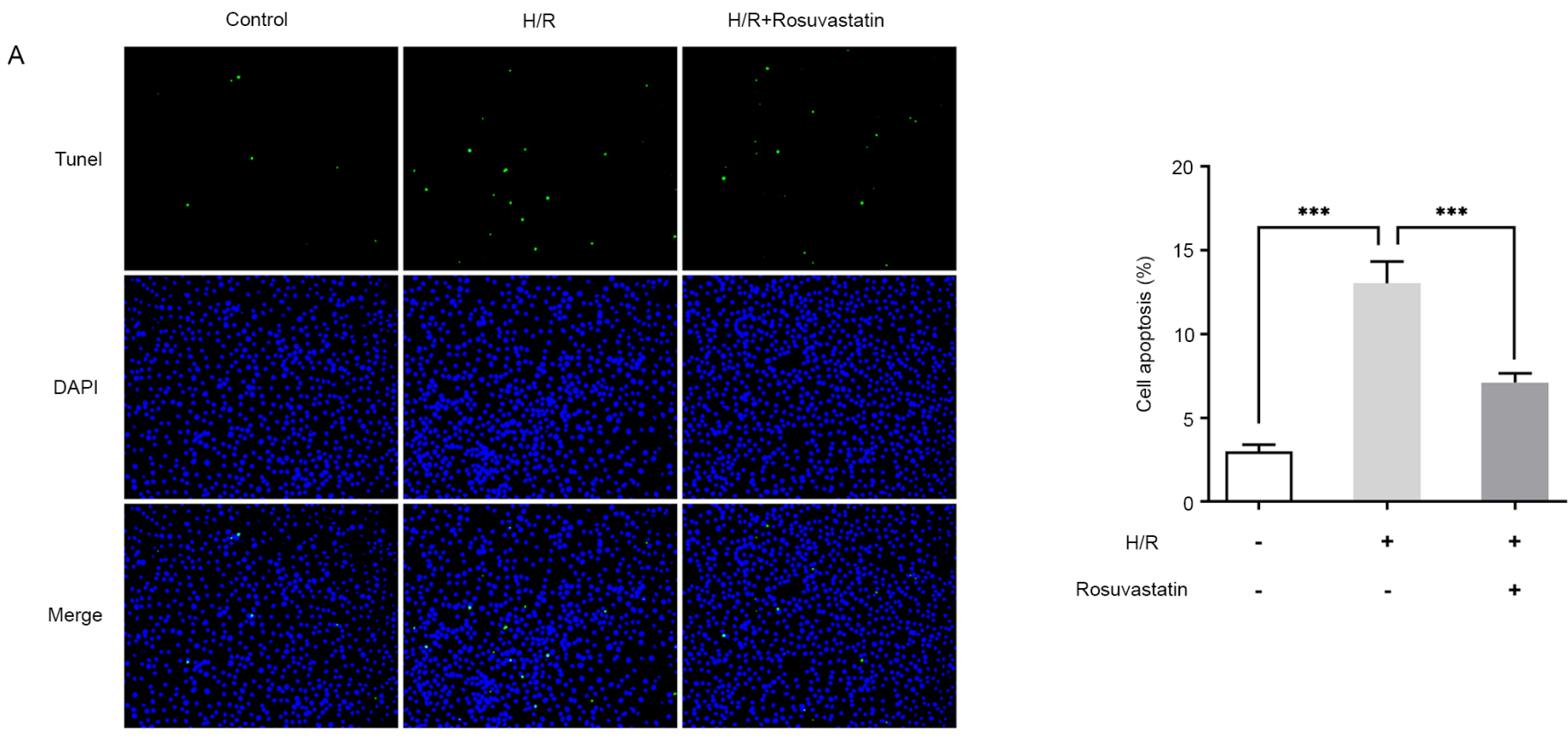

B
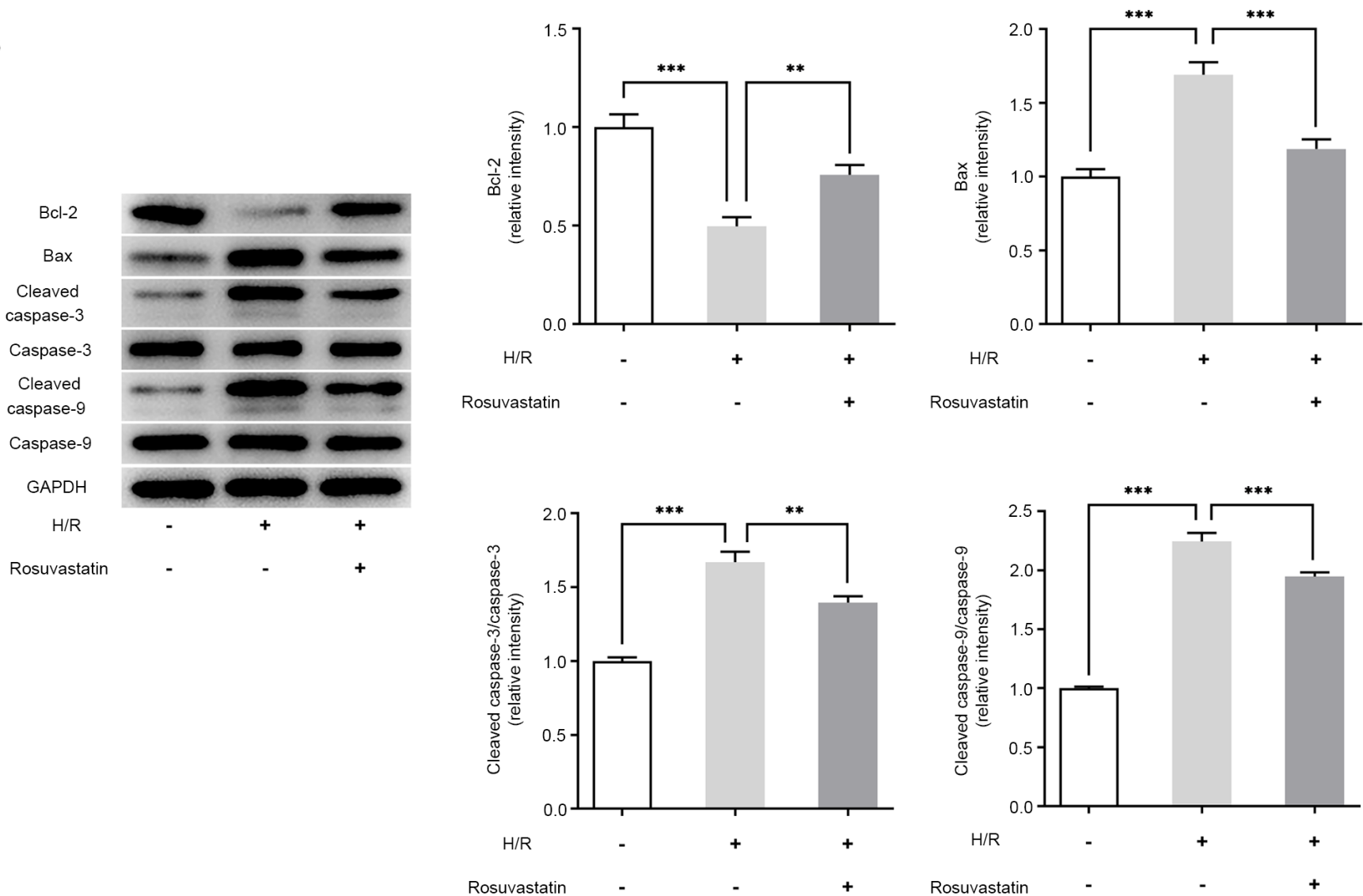

Figure 2. Rosuvastatin inhibits apoptosis of H/R-induced PC12 cells. (A) TUNEL assay detected cell apoptosis. Magnification, x200. (B) Western blotting detected apoptosis-associated proteins. ${ }^{* *} \mathrm{P}<0.01 ;{ }^{* * * *} \mathrm{P}<0.001$. H/R, hypoxia/reoxygenation.

Cell viability assay. Cell viability was measured using a Cell Counting Kit (CCK)-8 assay according to the manufacturer's instructions (Merck KGaA). Briefly, PC12 cells were seeded into 96 -well plates $\left(1 \times 10^{4}\right.$ cells/well). After the cells had reached $80 \%$ confluence, they were treated accordingly, and CCK-8 solution $(10 \mu \mathrm{l})$ was subsequently added to each well for an additional $4 \mathrm{~h}$ at $37^{\circ} \mathrm{C}$. The absorbance at $450 \mathrm{~nm}$ was measured with a microplate reader (Biotek Instruments, Inc.). Cell viability was expressed as a percentage of the control group cells.

Lactate dehydrogenase $(\mathrm{LDH})$ release assay. $\mathrm{LDH}$ detection is usually performed for evaluating cytotoxicity. A commercial LDH assay kit was purchased from Nanjing Jiancheng 


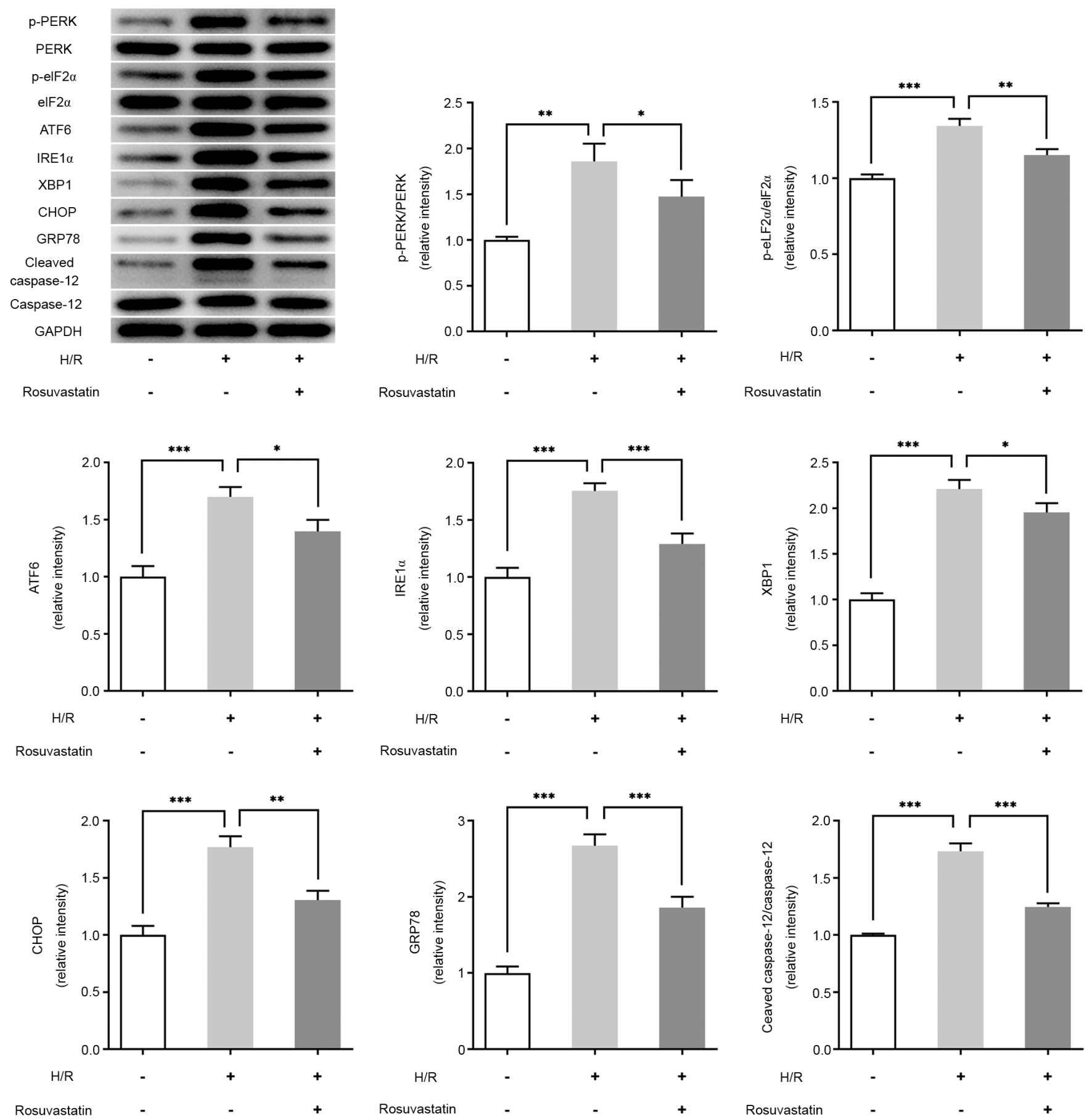

Figure 3. Rosuvastatin inhibits ERS levels in H/R-induced PC12 cells. Western blotting detected ERS-associated proteins. ${ }^{*} \mathrm{P}<0.05 ;{ }^{* * *} \mathrm{P}<0.01 ;{ }^{* * * *} \mathrm{P}<0.001 . \mathrm{H} / \mathrm{R}$, hypoxia/reoxygenation; ERS, endoplasmic reticulum stress; p-, phosphorylated-; CHOP, C/EBP homologous protein; eIF2 $\alpha$, eukaryotic initiation factor $2 \alpha$; ATF6, activating transcription factor 6; IRE1 $\alpha$, inositol-requiring transmembrane kinase/endoribonuclease $1 \alpha$; XBP1, X box binding protein 1 .

Bioengineering Institute (Nanjing, China) and the LDH assay was performed strictly according to the manufacturer's instructions. The absorbance of each sample was detected at $440 \mathrm{~nm}$ with a microplate reader. The percentage of cell death was calculated according to the following formula: Viability $(\%)=\left(\mathrm{OD}_{\text {Treatment }}-\mathrm{OD}_{\text {Treatment blank }}\right) /\left(\mathrm{OD}_{\text {Max LDH activity }}-\mathrm{OD}_{\text {Max LDH }}\right.$ activity blank $) \times 100 \%$.

TUNEL assay. The extent of apoptosis was detected by TUNEL assay (Promega Corporation), according to the manufacturer's instructions. The PC12 cells $\left(1 \times 10^{4}\right.$ cells/well) were incubated in 6-well plates for $24 \mathrm{~h}$. Cells were subsequently fixed using $4 \%$ paraformaldehyde for $30 \mathrm{~min}$ at $37^{\circ} \mathrm{C}$, and washed three times with cold PBS. The TUNEL reaction mix was added to the cells and incubated at $37^{\circ} \mathrm{C}$ for $1 \mathrm{~h}$. Cells were subsequently rinsed with PBS, streptavidin-HRP was added and the mixture was incubated for $1 \mathrm{~h}$ in the dark. Finally, DAB solution was added to develop the color reaction. Cells were observed under a light microscope (magnification, x200), and images were captured. The number of apoptotic cells was calculated as the mean proportion of positive cells out of the total number of cells in six fields of view per slide. 
A

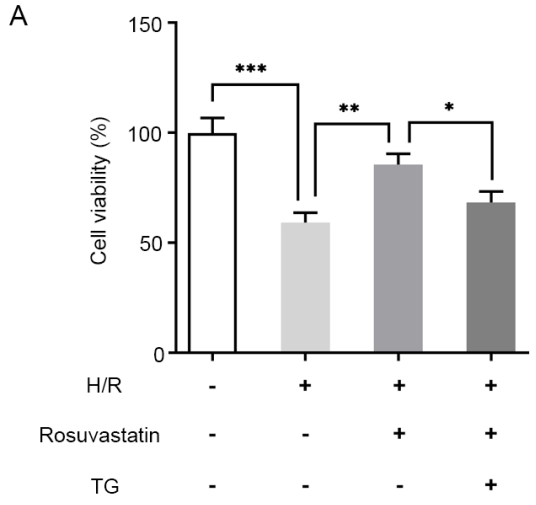

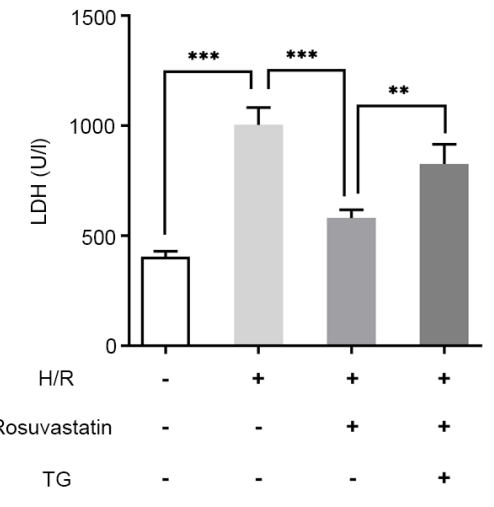

C

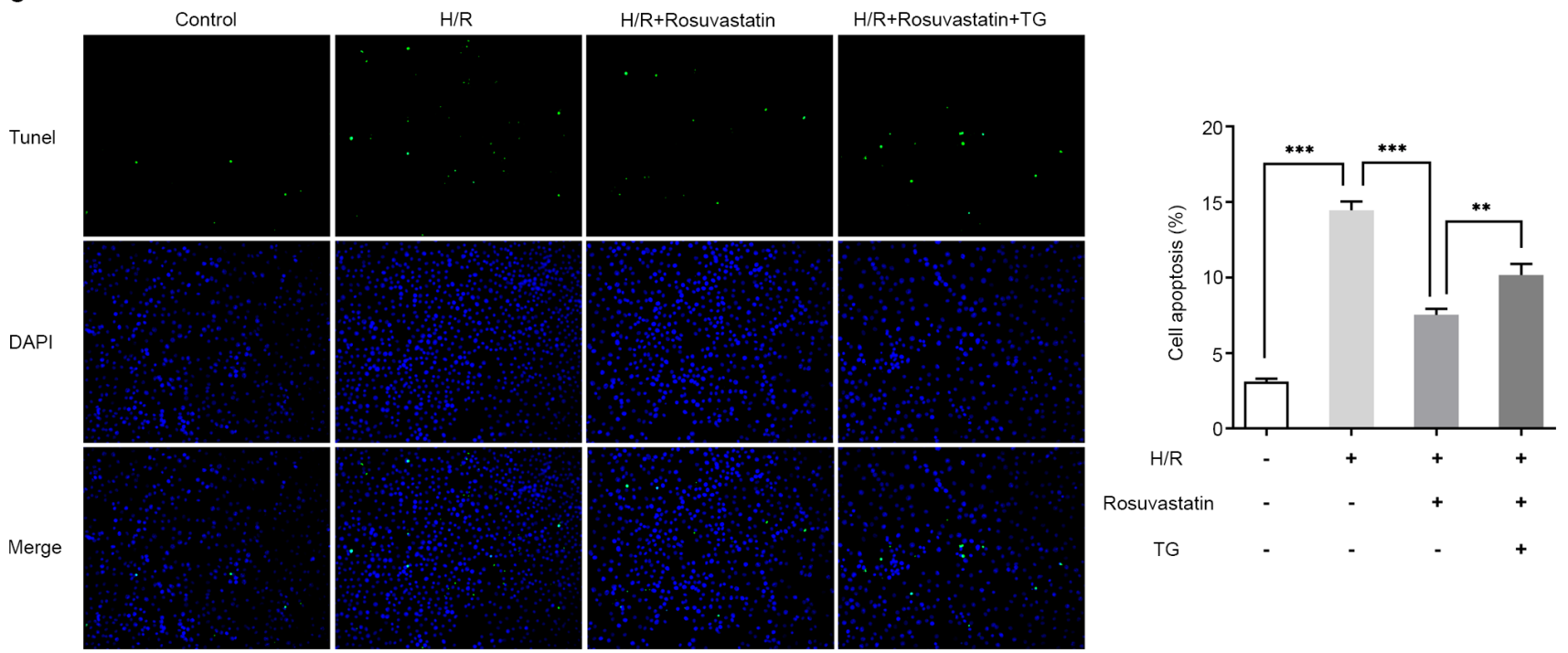

Figure 4. TG reverses the protective effect of rosuvastatin on the cell viability of H/R-induced PC12 cell. (A) Cell Cycle Kit- 8 detected cell viability. (B) LDH kit detected cytotoxicity. (C) TUNEL assay detected cell apoptosis. Magnification, $\mathrm{x} 200 .{ }^{*} \mathrm{P}<0.05 ;{ }^{* *} \mathrm{P}<0.01$; ${ }^{* * *} \mathrm{P}<0.001$. TG, thapsigargin; H/R, hypoxia/reoxygenation; LDH, lactate dehydrogenase.

Western blot analysis. PC12 cells were washed three times with ice-cold phosphate buffered saline, and lysis buffer (Merck $\mathrm{KGaA}$ ) was added to the cells for $30 \mathrm{~min}$ to isolate the total protein. Protein concentration was determined using a bicinchoninic acid assay protein assay kit (Bio-Rad Laboratories, Inc.). Proteins (25 $\mu \mathrm{g}$ /lane) were separated using 10\% SDS-PAGE gel and transferred to a polyvinylidene fluoride membrane, prior to blocking with $5 \%$ non-fat milk in Tris-buffered saline $/ 0.10 \%$ Tween-20 at room temperature for $2 \mathrm{~h}$. The membranes were then incubated with the following primary antibodies at $4^{\circ} \mathrm{C}$ overnight (all antibodies were purchased from Abcam): Anti-Bcl-2 (1:1,000; cat. no. ab182858), anti-Bax (1:1,000; cat. no. ab182733), anti-cleaved caspase-3 (1:1,000; cat. no. ab32042), anti-cleaved caspase-9 (1:1,000; cat. no. ab2324), anti-caspase-3 (1:1,000; cat. no. ab32351), anti-caspase-9 (1:1,000; cat. no. ab32539), anti-phosphorylated (p)-protein kinase R-like endoplasmic reticulum kinase (PERK; 1:1,000; cat. no. ab192591), anti-p-eukaryotic initiation factor $2 \alpha$ (eIF2 $\alpha ; 1: 1,000$; cat. no. ab32157), anti-eIF2 $\alpha(1: 1,000$; cat. no. ab169528), anti-PERK (1:1,000; cat. no. ab229912), anti-GRP78 (1:1,000; cat. no. ab21685), anti-activating transcription factor 6 (ATF6; 1:1,000; cat. no. ab227830), anti-inositol-requiring transmembrane kinase/endoribonuclease
$1 \alpha$ (IRE1 $\alpha ; 1: 1,000$; cat. no. ab37073), anti-X-box binding protein 1 (XBP1; 1:1,000; cat no. ab37152), anti-C/EBP homologous protein (CHOP; 1:1,000; cat. no. ab11419) and anti-GAPDH (1:1,000; cat. no. ab8245), and anti-cleaved caspase-12/caspase 12 (1:1,000; cat. no. \#2202; Cell Signaling Technology, Inc.). After washing with Tris-buffered saline/0.10\% Tween-20, the blots were incubated with Goat Anti-Mouse IgG H\&L (Alexa Fluor $^{\circledR}$ 488; 1:5,000; cat. no. ab150113; Abcam) for $2 \mathrm{~h}$ at room temperature. Signals were visualized with enhanced chemiluminescence (Beyotime Institute of Biotechnology), and ImageJ software (Version1.8.0; National Institutes of Health) was used for semi-quantification of the blots.

Statistical analysis. Data are expressed as the mean \pm SD. Comparisons among multiple groups were analyzed using a one-way ANOVA followed by a Tukey's post hoc test. $\mathrm{P}<0.05$ was considered to indicate a statistically significant difference.

\section{Results}

Rosuvastatin inhibits decreased cellular activity in H/R-induced PC12 cells. The chemical structure of rosuvastatin is shown in Fig. 1A. PC12 cells were induced by 

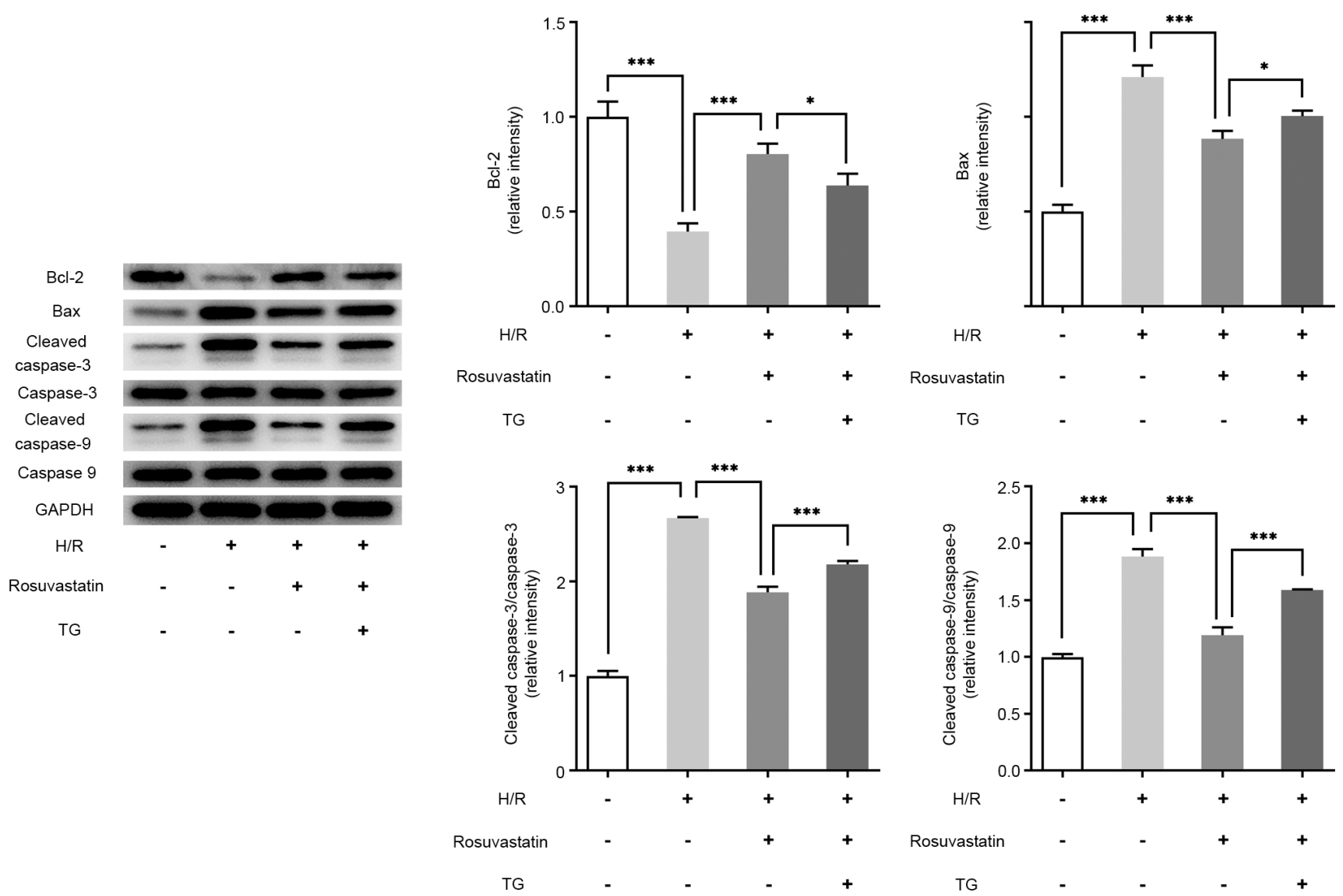

Figure 5. TG reverses the protective effect of rosuvastatin on the apoptosis of H/R-induced PC12 cell. Western blotting detected the apoptosis-associated proteins. ${ }^{*} \mathrm{P}<0.05 ;{ }^{* * *} \mathrm{P}<0.001$. TG, thapsigargin; $\mathrm{H} / \mathrm{R}$, hypoxia/reoxygenation.

rosuvastatin at different concentrations $(0,0.01,0.1,1$ and $10 \mu \mathrm{M})$. The results of the CCK-8 assay demonstrated that rosuvastatin exerted no significant effect on the viability of PC12 cells (Fig. 1B). Subsequently, CCK-8 assay was used to investigate the effect of rosuvastatin on the viability of H/R-induced PC12 cells, and it was observed that, with an increase in rosuvastatin concentration, the viability of H/R-induced PC12 cells was significantly increased (Fig. 1C). LDH kit assay was then performed for the detection of cytotoxicity, and these results showed that rosuvastatin led to a marked decrease in the cytotoxicity of H/R-induced PC12 cells (Fig. 1D). Taken together, the results from these assays revealed that rosuvastatin treatment led to decreased cellular activity in H/R-induced PC12 cells. In addition, $1 \mu \mathrm{M}$ Rosuvastatin had significant effects on cell viability and cell LDH release, thus, $1 \mu \mathrm{M}$ rosuvastatin was selected as the concentration for subsequent experiments.

Rosuvastatin inhibits apoptosis of H/R-induced PC12 cells. Apoptosis was detected using a TUNEL assay. Compared with the control group, apoptosis was found to be significantly increased following $\mathrm{H} / \mathrm{R}$ induction (Fig. 2A), accompanied by an increased expression of the Bax, cleaved caspase-3, cleaved caspase-9 proteins, whereas the expression level of the Bcl-2 protein was decreased (Fig. 2B). Compared with the H/R group, the apoptotic rate of the H/R + rosuvastatin group was decreased, accompanied by a decreased expression of cleaved caspase-3, cleaved caspase- 9 , and an increased expression of
Bcl-2. These results showed that rosuvastatin is able to inhibit apoptosis in H/R-induced PC12 cells.

Rosuvastatin inhibits ERS levels in H/R-induced PC12 cells. In order to detect the effect of rosuvastatin on ERS levels in H/R-induced PC12 cells, western blot analysis was used to investigate the expression levels of ERS-associated proteins. Compared with the control group, the expression levels of p-PERK, p-eIF2 $\alpha$, ATF6, IRE1 $\alpha$, XBP1, CHOP, GRP78 and cleaved caspase-12 were significantly increased in the H/R group. Further administration of rosuvastatin, however, reversed the expression levels of p-PERK, p-eIF2 $\alpha$, ATF6, IRE1 $\alpha$, XBP1, CHOP, GRP78, CHOP and cleaved caspase-12 (Fig. 3).

$T G$ reverses the protective effects of rosuvastatin on $H / R$-induced PC12 cell injury. Subsequently, the mechanism of rosuvastatin action on H/R-induced PC12 cells by adding the ERS activator, thapsigargin (TG), was investigated. To this end, the cells were divided into control, H/R, H/R + rosuvastatin and $H / R+$ rosuvastatin + TG groups. The results of CCK-8 assay showed that, compared with the H/R + rosuvastatin group, the cell survival rate was significantly reversed following TG addition (Fig. 4A). The results of the LDH kit assay revealed that, compared with the H/R + rosuvastatin group, the cytotoxicity of the H/R + rosuvastatin $+T G$ group was significantly increased (Fig. 4B). The results from the TUNEL assay experiments revealed that TG administration was able 


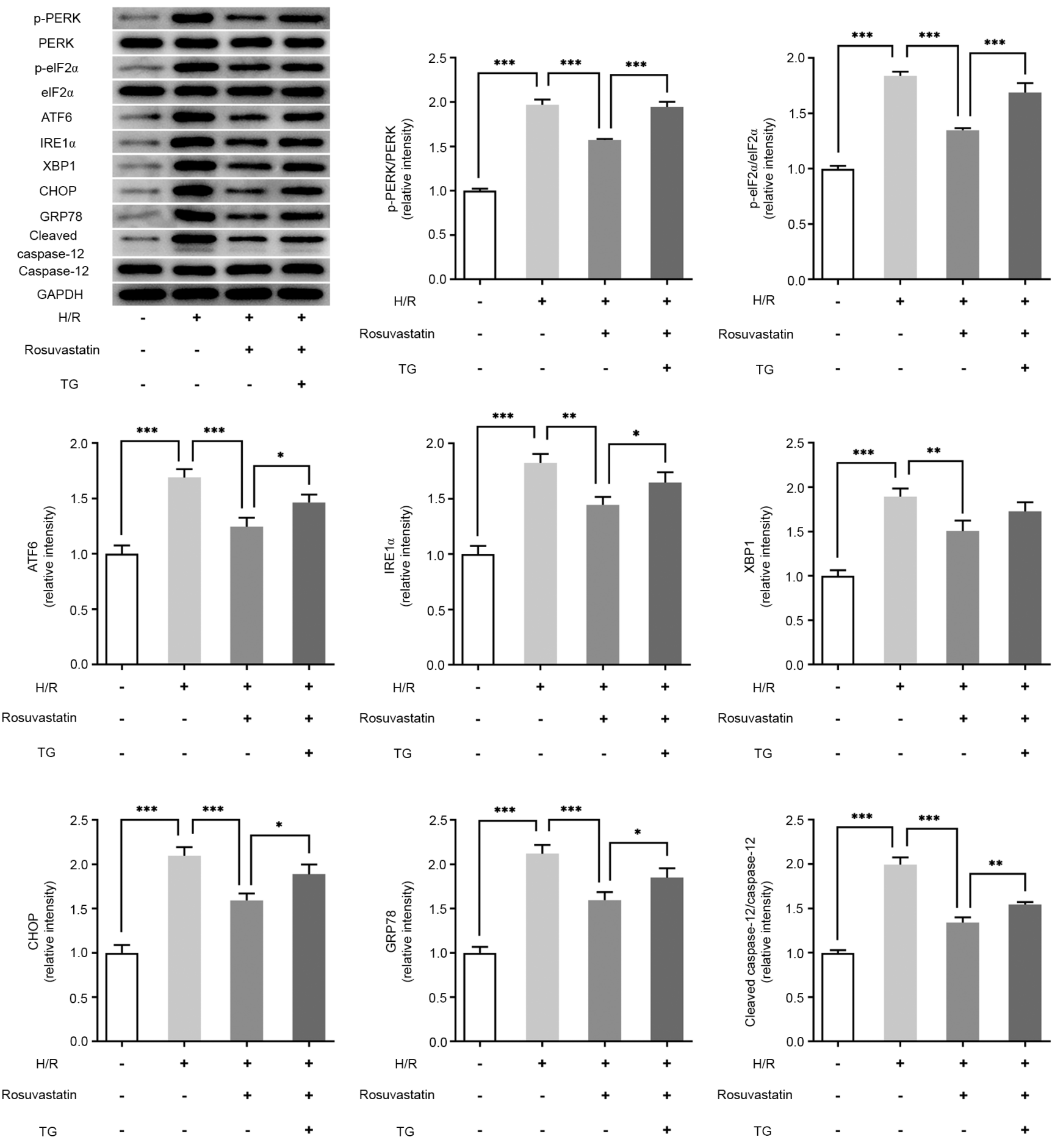

Figure 6. TG reverses the protective effect of rosuvastatin on ERS of H/R-induced PC12 cell. Western blotting detected the ERS-associated proteins. "P<0.05; ${ }^{* *} \mathrm{P}<0.01 ;{ }^{* * *} \mathrm{P}<0.001 . \mathrm{H} / \mathrm{R}$, hypoxia/reoxygenation; ERS, endoplasmic reticulum stress; $\mathrm{p}-$, phosphorylated-; CHOP, C/EBP homologous protein; eIF2 $\alpha$, eukaryotic initiation factor $2 \alpha$; ATF6, activating transcription factor 6; IRE1 $\alpha$, inositol requiring transmembrane kinase/endoribonuclease $1 \alpha$; XBP1, X-box binding protein 1.

to significantly reverse the apoptotic rate of H/R-induced PC12 cells inhibited by rosuvastatin (Fig. 4C). Subsequently, the expression levels of apoptosis-associated proteins were investigated, and the trend in expression level changes was consistent with those of the TUNEL assay (Fig. 5). In addition, the expression levels of ERS-associated proteins were also investigated, and these experiments showed that, compared with the H/R + rosuvastatin group, the expression levels of p-PERK, p-eIF2 $\alpha$, ATF6, IRE1 $\alpha$, XBP1, CHOP, GRP78 and cleaved caspase-12 were significantly increased (Fig. 6). Taken together, the aforementioned experimental results showed that TG reversed the protective effect of rosuvastatin on H/R-induced PC12 cell injury.

\section{Discussion}

PC12 cells are a differentiated cell line of pheochromocytoma in the adrenal medulla of rats. PC12 cells have the 


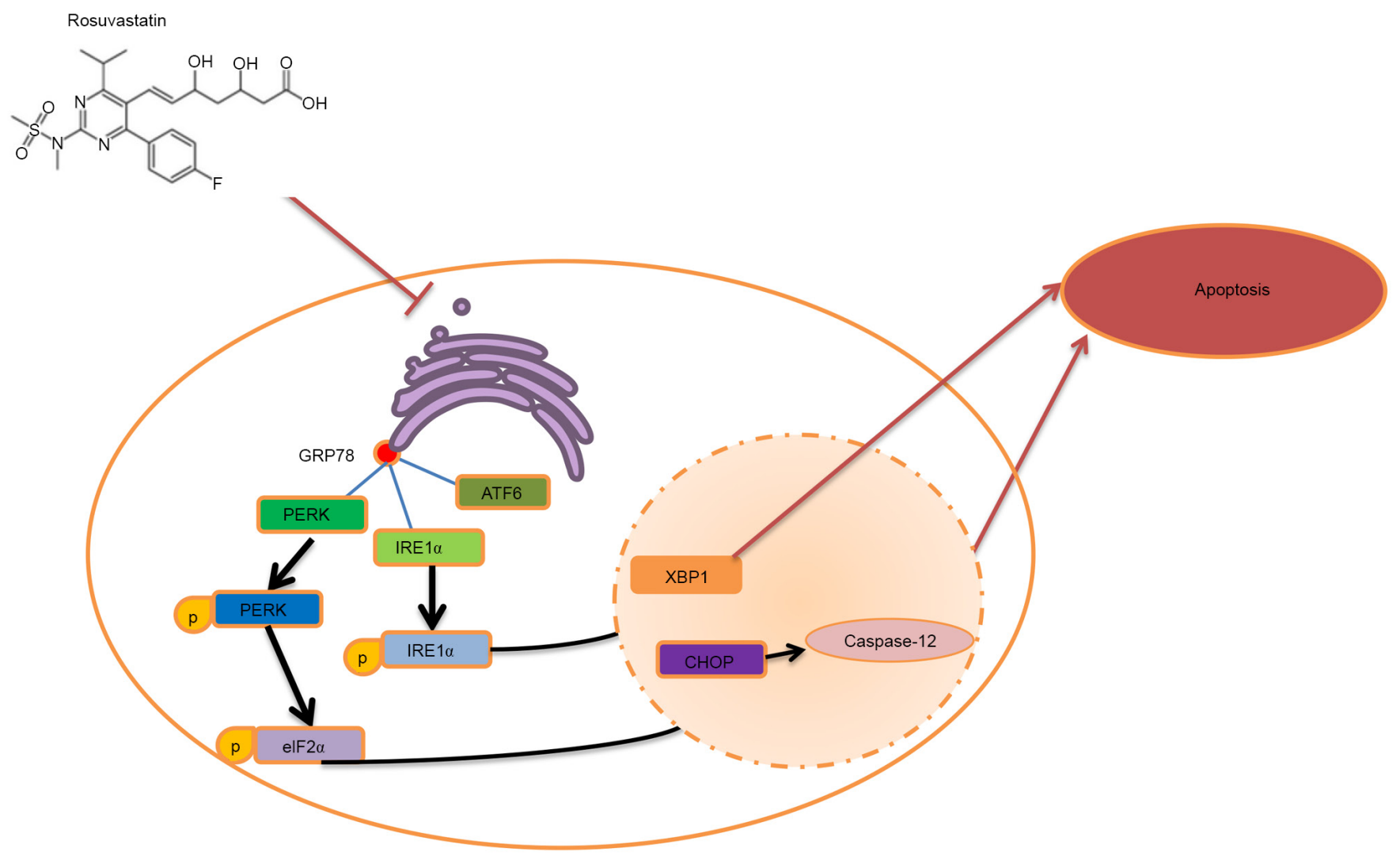

Figure 7. Mechanism of action. p-, phosphorylated-; CHOP, C/EBP homologous protein; eIF2 $\alpha$, eukaryotic initiation factor $2 \alpha$; ATF6, activating transcription factor 6; IRE1 $\alpha$, inositol requiring transmembrane kinase/endoribonuclease $1 \alpha ; \mathrm{XBP} 1, \mathrm{X}$-box binding protein 1 .

general characteristics of neuroendocrine cells and are widely used in neurophysiological and pharmacological studies due to their ability of passage. In addition, a study has used hypoxia-reoxygenation-induced PC12 cells to form a model of cerebral ischemia reperfusion injury model, which has been recognized as a model (20). Therefore, in the present study, PC12 cells were induced to establish a damage model through $\mathrm{H} / \mathrm{R}$ and carried out relevant experiments.

ERS mediates three different signaling pathways at both the transcriptional and translational levels, predominantly through ER transmembrane proteins, namely, the three unfolded protein response signaling receptor molecules, IRE-1, PERK and activating transcription factor 6 (21). These three signaling molecules are normally inactive. When ERS occurs, heat shock protein family A (Hsp70) member 5 (GRP78) is isolated from these signal receptor molecules, which assists in the process of enabling unfolded or misfolded proteins to recover their correct conformation, and activating these signal receptor molecules, affecting the downstream CHOP levels (22). Previous studies have shown that the inhibition of ERS-mediated apoptosis leads to a decrease in CIRI-induced injury $(12,23,24)$. Therefore, the ER should serve an important role in the development of CIRI. In the present study, a damage model of PC12 cells induced by $\mathrm{H} / \mathrm{R}$ was established. It was found that the expression levels of ERS-associated proteins were significantly increased, and the cell apoptotic rate was also increased, indicating that ERS-induced apoptosis has an important role in $\mathrm{H} / \mathrm{R}$-induced cell damage.
A previous study reported that rosuvastatin is able to alleviate myocardial IR injury by upregulating miR-17-3s-mediated autophagy (25). Rosuvastatin protects isolated hearts from IR injury through the Akt/GSK-3 $\beta$ signaling axis, the metabolic environment and mitochondrial permeability transition hole (26). Pretreatment with rosuvastatin has been shown to decrease oxidative stress and the inflammatory response associated with focal CIRI in rats (16). In addition, rosuvastatin has been demonstrated to have an important neuroprotective role in animal models of CIRI through decreasing inflammatory damage, regulating thrombosis and improving endothelial cell function. Furthermore, rosuvastatin has been widely recommended for primary and secondary defense treatment of CIRI (27-29).

Rosuvastatin alleviates endothelial cell apoptosis and atherosclerosis of $\mathrm{ApoE}^{-/-}$mice by inhibiting ERS (19). It has also been shown that rosuvastatin may protect endothelial cells from hyperglycemia-induced damage by decreasing ERS (18). Rosuvastatin and simvastatin attenuate cisplatin-induced cardiotoxicity through the disruption of ERS-mediated apoptotic death in rats via targeting the ER-chaperone GRP78 and calpain-1 pathways (30). However, the regulatory effect of rosuvastatin on ERS in CIRI has not yet been reported, to the best of our knowledge. In the present study, it was shown that rosuvastatin could inhibit the cell activity decline, decrease the cytotoxicity and inhibit the apoptotic rate and ERS level of H/R-induced PC12 cells. By adding the ERS activator TG, the protective effect of rosuvastatin on H/R-induced PC12 cell injury was found 
to be significantly reversed. The aforementioned findings suggested that rosuvastatin may protect PC12 cells from H/R-induced injury through inhibiting apoptosis induced by ERS.

In conclusion, the present study has shown that rosuvastatin protects PC12 cells from H/R-induced injury by inhibiting ERS-induced apoptosis (Fig. 7). The findings may provide a theoretical basis for the clinical treatment of CIRI with rosuvastatin.

\section{Acknowledgements}

Not applicable.

\section{Funding}

The present study was funded by Hunan Province Traditional Chinese Medicine Research Project (grant no. 202015) and Scientific Research Project of Hunan Provincial Health and Family Planning Commission (grant no. 20200308).

\section{Availability of data and materials}

The datasets used and/or analyzed during the present study are available from the corresponding author on reasonable request.

\section{Authors' contributions}

$\mathrm{ZZ}$ and YG wrote the manuscript and analyzed the data. LL and JY performed the experiments and supervised the study. YG searched the literature and revised the manuscript for important intellectual content. ZZ and YG confirm the authenticity of all the raw data. All authors have read and approved the final manuscript.

\section{Ethics approval and consent to participate}

Not applicable.

\section{Patient consent for publication}

Not applicable.

\section{Competing interests}

The authors declare that they have no competing interests.

\section{References}

1. Wang M, Wang J, Liu Z, Guo X, Wang N, Jia N, Zhang Y and Yuan J: Effects of intermedin on autophagy in cerebral ischemia/reperfusion injury. Neuropeptides 68: 15-21, 2018.

2. Deng Y, Zou W, Chen G, Shangguan S, Zhou F, Jiang W and Li X: Comparative studies on the effects of different doses of atorvastatin combined with aspirin on inflammatory cytokines and carotid plaques in patients with ischemic cerebrovascular disease. Int J Neurosci 129: 1133-1138, 2019.

3. Wu MY, Yiang GT, Liao WT, Tsai AP, Cheng YL, Cheng PW, Li CY and Li CJ: Current mechanistic concepts in ischemia and reperfusion injury. Cell Physiol Biochem 46: 1650-1667, 2018.
4. Sun MS, Jin H, Sun X, Huang S, Zhang FL, Guo ZN and Yang Y: Free radical damage in ischemia-reperfusion injury: An obstacle in acute ischemic stroke after revascularization therapy. Oxid Med Cell Longev 2018: 3804979, 2018.

5. Xu F, Ma R, Zhang G, Wang S, Yin J, Wang E, Xiong E, Zhang Q and Li Y: Estrogen and propofol combination therapy inhibits endoplasmic reticulum stress and remarkably attenuates cerebral ischemia-reperfusion injury and OGD injury in hippocampus. Biomed Pharmacother 108: 1596-1606, 2018.

6. Lin YW, Chen TY, Hung CY, Tai SH, Huang SY, Chang CC Hung HY and Lee EJ: Melatonin protects brain against ischemia/reperfusion injury by attenuating endoplasmic reticulum stress. Int J Mol Med 42: 182-192, 2018.

7. Feng D, Wang B, Wang L, Abraham N, Tao K, Huang L, Shi W, Dong Y and Qu Y: Pre-ischemia melatonin treatment alleviated acute neuronal injury after ischemic stroke by inhibiting endoplasmic reticulum stress-dependent autophagy via PERK and IRE1 signalings. J Pineal Res 62, 2017.

8. Nakka VP, Gusain A and Raghubir R: Endoplasmic reticulum stress plays critical role in brain damage after cerebral ischemia/reperfusion in rats. Neurotox Res 17: 189-202, 2010.

9. Xin Q, Ji B, Cheng B, Wang C, Liu H, Chen X, Chen J and Bai B: Endoplasmic reticulum stress in cerebral ischemia. Neurochem Int 68: 18-27, 2014

10. Ishige K, Osada N, Kosuge Y and Ito Y: Involvement of endoplasmic reticulum stress in neurodegeneration after transient global ischemia-reperfusion. Nihon Yakurigaku Zasshi 142: 9-12, 2013 (In Japanese).

11. Gong L, Tang Y, An R, Lin M, Chen L and Du J: RTN1-C mediates cerebral ischemia/reperfusion injury via ER stress and mitochondria-associated apoptosis pathways. Cell Death Dis 8: e3080, 2017.

12. Feng SQ, Zong SY, Liu JX, Chen Y, Xu R, Yin X, Zhao R, Li Y and Luo TT: VEGF antagonism attenuates cerebral ischemia/reperfusion-induced injury via inhibiting endoplasmic reticulum stress-mediated apoptosis. Biol Pharm Bull 42: 692-702, 2019.

13. Gutiérrez-Vargas JA, Cespedes-Rubio A and Cardona-Gómez GP: Perspective of synaptic protection after post-infarction treatment with statins. J Transl Med 13: 118, 2015.

14. Cortese F, Gesualdo M, Cortese A, Carbonara S, Devito F, Zito A, Ricci G, Scicchitano P and Ciccone MM: Rosuvastatin: Beyond the cholesterol-lowering effect. Pharmacol Res 107: 1-18, 2016.

15. Liu Y, Yang H, Jia G, Li L, Chen H, Bi J and Wang C: The synergistic neuroprotective effects of combined rosuvastatin and resveratrol pretreatment against cerebral ischemia/reperfusion injury. J Stroke Cerebrovasc Dis 27: 1697-1704, 2018.

16. Ma M, Uekawa K, Hasegawa Y, Nakagawa T, Katayama T, Sueta D, Toyama K, Kataoka K, Koibuchi N, Kuratsu J and Kim-Mitsuyama S: Pretreatment with rosuvastatin protects against focal cerebral ischemia/reperfusion injury in rats through attenuation of oxidative stress and inflammation. Brain Res 1519: 87-94, 2013.

17. Engelhorn T, Doerfler A, Heusch G and Schulz R: Reduction of cerebral infarct size by the AT1-receptor blocker candesartan, the HMG-CoA reductase inhibitor rosuvastatin and their combination. An experimental study in rats. Neurosci Lett 406: 92-96, 2006.

18. Xu JZ, Chai YL and Zhang YL: Effect of rosuvastatin on high glucose-induced endoplasmic reticulum stress in human umbilical vein endothelial cells. Genet Mol Res 15, 2016.

19. Geng J, Xu H, Fu W, Yu X, Xu G, Cao H, Lin G and Sui D: Rosuvastatin protects against endothelial cell apoptosis in vitro and alleviates atherosclerosis in $\mathrm{ApoE}^{-/}$mice by suppressing endoplasmic reticulum stress. Exp Ther Med 20: $550-560,2020$.

20. Chen $\mathrm{H}$ and Li $\mathrm{X}$ : LncRNA ROR is involved in cerebral hypoxia/reoxygenation-induced injury in PC12 cells via regulating miR-135a-5p/ROCK1/2. Am J Transl Res 11: 6145-6158, 2019.

21. Oakes SA and Papa FR: The role of endoplasmic reticulum stress in human pathology. Annu Rev Pathol 10: 173-194, 2015.

22. Zhang $\mathrm{K}$ and Kaufman RJ: The unfolded protein response: A stress signaling pathway critical for health and disease. Neurology 66 (2 Suppl 1): S102-S109, 2006.

23. Han ZW, Chang YC, Zhou Y, Zhang H, Chen L, Zhang Y, Si JQ and Li L: GPER agonist G1 suppresses neuronal apoptosis mediated by endoplasmic reticulum stress after cerebral ischemia/reperfusion injury. Neural Regen Res 14: 1221-1229, 2019. 
24. Zhong CJ, Chen MM, Lu M, Ding JH, Du RH and $\mathrm{Hu} G$ : Astrocyte-specific deletion of Kir6.1/K-ATP channel aggravates cerebral ischemia/reperfusion injury through endoplasmic reticulum stress in mice. Exp Neurol 311: 225-233, 2019.

25. Wang X, Chen J and Huang X: Rosuvastatin attenuates myocardial ischemia-reperfusion injury via upregulating miR-17-3p-mediated autophagy. Cell Reprogram 21: 323-330, 2019.

26. Vélez DE, Mestre-Cordero VE, Hermann R, Perego J, Harriet S, Fernandez-Pazos MLM, Mourglia J and Marina-Prendes MG: Rosuvastatin protects isolated hearts against ischemia-reperfusion injury: Role of Akt-GSK-3 $\beta$, metabolic environment, and mitochondrial permeability transition pore. J Physiol Biochem 76: 85-98, 2020

27. Griffin JM, Kho D, Graham ES, Nicholson LF and O'Carroll SJ: Statins inhibit fibrillary $\beta$-amyloid induced inflammation in a model of the human blood brain barrier. PLoS One 11: e0157483, 2016

28. Rodriguez-Perea AL, Gutierrez-Vargas J, Cardona-Gómez GP, Guarin CJ, Rojas M and Hernández PA: Atorvastatin modulates regulatory $\mathrm{T}$ cells and attenuates cerebral damage in a model of transient middle cerebral artery occlusion in rats. J Neuroimmune Pharmacol 12: 152-162, 2017.
29. Sohn HM, Hwang JY, Ryu JH, Kim J, Park S, Park JW and Han SH: Simvastatin protects ischemic spinal cord injury from cell death and cytotoxicity through decreasing oxidative stress: In vitro primary cultured rat spinal cord model under oxygen and glucose deprivation-reoxygenation conditions. J Orthop Surg Res 12: 36, 2017.

30. Saleh DO, Mansour DF and Mostafa RE: Rosuvastatin and simvastatin attenuate cisplatin-induced cardiotoxicity via disruption of endoplasmic reticulum stress-mediated apoptotic death in rats: Targeting ER-chaperone GRP78 and calpain-1 pathways. Toxicol Rep 7: 1178-1186, 2020

(1) () This work is licensed under a Creative Commons Attribution-NonCommercial-NoDerivatives 4.0 International (CC BY-NC-ND 4.0) License. 